UCRL-ID-130783

\title{
Interfacial Effects in Multilayers
}

T.W. Barbee, Jr.

June 28, 1999

U.S. Department of Energy

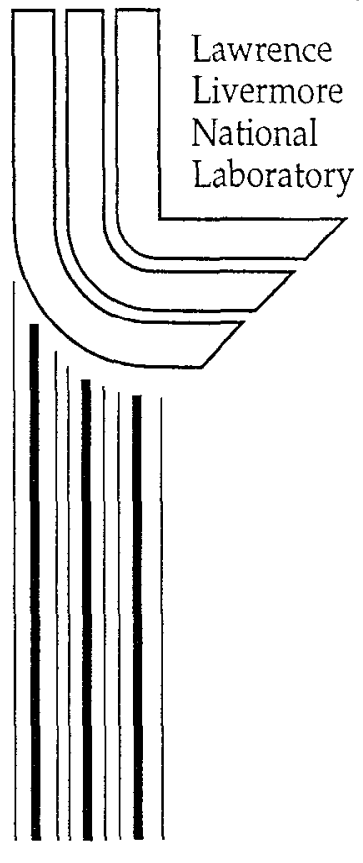




\section{DISCLAIMER}

This document was prepared as an account of work sponsored by an agency of the United States Government. Neither the United States Government nor the University of California nor any of their employees, makes any warranty, express or implied, or assumes any legal liability or responsibility for the accuracy, completeness, or usefulness of any information, apparatus, product, or process disclosed, or represents that its use would not infringe privately owned rights. Reference herein to any specific commercial product, process, or service by trade name, trademark, manufacturer, or otherwise, does not necessarily constitute or imply its endorsement, recommendation, or favoring by the United States Government or the University of California. The views and opinions of authors expressed herein do not necessarily state or reflect those of the United States Government or the University of California, and shall not be used for advertising or product endorsement purposes.

This report has been reproduced directly from the best available copy.

Available to DOE and DOE contractors from the Office of Scientific and Technical Information

P.O. Box 62, Oak Ridge, TN 37831

Prices available from (615) 576-8401, FTS 626-8401

Available to the public from the

National Technical Information Service

U.S. Department of Commerce

5285 Port Royal Rd.,

Springfield, VA 22161 


\title{
INTERFACIAL, EFFECTS IN MULTILAYERS
}

\author{
TROY W. BARBEE, JR. \\ Lawrence Livermore National Laboratory \\ Livermore, CA 94550, USA
}

\section{INTRODUCTION}

There are many physical characterization approaches ${ }^{1}$ which evaluate a limited set of structural elements in multilayers: they study a single interface ${ }^{2,3,4}$; they study a single layer of material; they study a very small sample of a multilayer ${ }^{5,6}$. On a broader basis, the interference phenomena on which the performance of $x$-ray optic multilayers is based integrates over the full area/volume ${ }^{7,8,9}$ of the multilayer illuminated. In order to gain understanding of the impact of imperfections on multilayer performance it is necessary to develop an experimental approach that provides detailed information about the effects of interfaces in the multilayer obtained when the multilayer is being applied in a manner directly related to application. Additionally, it is also of interest to determine the breadth of application of any such experimental approach to the general study of interfaces in solids.

The primary goal in this research was to develop an experimental methodology to quantitatively characterize both the physical and electronic characteristics of interfaces in multilayer structures The approach was to fabricate multilayers from three elements so that one monolayer or less thick "marker layers" were selectively deposited on a given set interfaces in the multilayer. These "marker layers" could then interrogated by scattering and fluorescence techniques for their distribution, for their atomic arrangements relative to the thicker layers and for their clectronic state at the interfaces as affected by the thicker layer materials. WC/C multilayers with one monolayer $(2.33 \AA)$ of tantalum at the WC on $\mathrm{C}$ and the $\mathrm{C}$ on WC interfaces were fabricated and studied. Ta was selected as the marker layer material as its $\mathrm{L}_{3}$ absorption edge is at $9879 \mathrm{eV}$, more than $300 \mathrm{eV}$ less than the $\mathrm{W}_{3}$ edge at $10200 \mathrm{eV}$. Reflectivities at $9850 \mathrm{eV}, 9879 \mathrm{eV}$ and $9950 \mathrm{eV}$ were measured: Ta layers standing wave fluorescence on the multilayer Bragg peak at these energies and fluorescence EXAFS of the Ta layers were also obtained. These results are modeled and the implications of the results for $\mathrm{x}$-ray optic structures and the study of buried interfaces in solids discussed.

\section{EXPERIMENTAL DESIGN AND SAMPLE FABRICATION}

It is well known that at the Bragg peak a $x$-ray standing wave ${ }^{10}$ is established in a periodic structure such as a multilayer. At fixed $x$-ray wavelengths this standing wave sweeps a distance of half the multilayer period through the multilayer as the Bragg peak is scanned in angle space. The nodes and anti-nodes of this standing wave field sweep over the interfaces and, in principle, provide a probe for sampling the interface structure. Samples in this study were designed to determine the effects of interface imperfection on mutlilayer performance using this characteristic of the diffraction process. The objective with these samples was to determine the impact of imperfection position on the two interfaces characteristic of multilayers on performance. A second objective was to extend the experiments demonstrate the capability to study atomic arrangements and electronic structure at interfaces.

In the following a set of samples with a monolayer thick marker layer deposited at specified interfaces that may be fluoresced by the standing wave field as it sweeps through the mutlilayer are described and experimental results obtained at the Stanford Synchrotron Radiation Laboratory BL - 10 reported. Two (No's 110 \& 111) special multilayers designed to use the standing wave to sample interface structure were fabricated and characterized. The multilayers were synthesized using standard magnetron sputter deposition techniques from carbon (C), tungsten carbide (WC) and tantalum (Ta) and had 60 periods of $32.28 \AA$ (110) and $32.78 \AA$ (111). The structures synthesized are:

\section{No. 110 - (111) Silicon Substrate/C/Ta/WC/C/Ta/WC/C/Ta/...../Ta/WC/C/Ambient \\ No. 111 - (111) Silicon Substrate/C/WC/Ta/C/WC/Ta/C/WC/Ta/...../WC/Ta/C/Ambient.}

The design layer thicknesses deposited are $\mathrm{t}_{\mathrm{C}}=15.9 \AA, \mathrm{t}_{\mathrm{WC}}=15.6 \AA$ and $\mathrm{t}_{\mathrm{Ta}}=2.33 \AA$. It is expected that the Ta will react with $1.25 \AA$ of the carbon to form $\mathrm{TaC}$ resulting in the observed multilayer periods. The selection of these materials was made on the basis of three materials properties. First, WC is the highest carbon containing compound in the W/C binary system so that compositionally abrupt and potentially smooth interfaces are expected for the WC/C system. Second, it was expected that the Ta would react with the $\mathrm{C}$ to form $\mathrm{TaC}$ which is isostructural with WC. Third, and most important, the $L_{3}$ absorption edges of Ta and $W$ are $9881 \mathrm{eV}$ and 10,207 eve respectively. This difference in the absorption edge energies of $326 \mathrm{eV}$ made possible at the Stanford Synchrotron Radiation Laboratory's BL - 10 scanning in angle of incidence of the first order Bragg peaks of these samples at photon energies below, at and above the $\mathrm{L}_{3}$ edge of $\mathrm{Ta}$ at $9881 \mathrm{eV}$. In these experiments reflectivity was measured at 
energies of 9850,9879 and $9950 \mathrm{eV}$. Total standing wave fluorescence was also measured by placing a detector above and parallel to the multilayer surface and monitoring the fluorescent intensity as a function of angle of incidence at the same energies. The tantalum $L$ lines will only be excited at energies at or above approximately 9881 $\mathrm{eV}$ so that scans as described above will enable assessment of the effects absorbers/imperfections at specific interfaces in a X-ray optic multilayer.

\section{EXPERIMENTAL RESULTS AND MODELING}

X-ray reflectivity and standing wave experiments on multilayer samples were performed on BL 10-2 at the Stanford Synchrotron Radiation Laboratory at extreme grazing angles of incidence ( 0 to 3 deg.) by scanning angle at fixed photon energy or by scanning photon energy at fixed angle of incidence. There are strongly absorbing white lines on the $L_{3,2}$ edges of Ta and $W$ which dramatically enhance their effect on the reflectivity at those energies. The $\mathrm{Ta} \mathrm{L}_{3}$ white line energy of $9879 \mathrm{eV}$ used in the remainder of this paper was determined by performing an energy scan (EXAFS) at fixed angle of incidence over the energy range $9780 \mathrm{eV}$ to $9980 \mathrm{eV}$ and detecting the total fluorescent signal as shown in Figure 1. Multilayer reflectivities for sample No's 110 and 111 were measured at incident x-ray energies of $9850 \mathrm{eV}, 9879 \mathrm{eV}$ and $9950 \mathrm{eV}$. These werc sclected as the Ta absorption increases in the order $9850-9950-9879 \mathrm{eV}$. The effect of Ta absorption could thus be determined at three mass absorption values resulting in a more reliable data set.

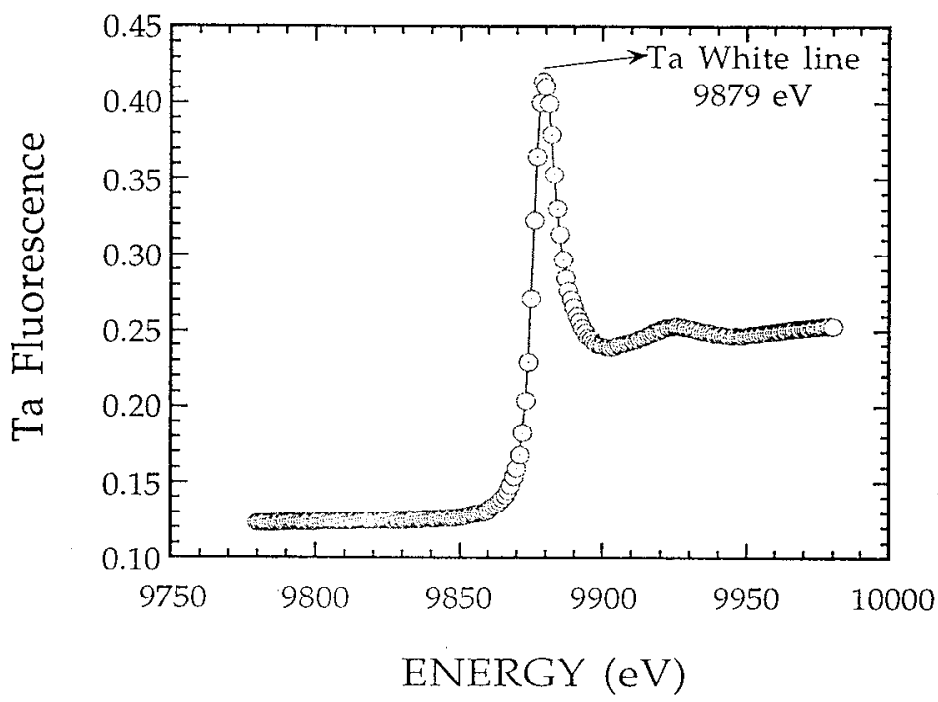

Figure 1. EXAFS scan of Sample 111 showing the $\mathrm{Ta} \mathrm{L}_{3}$ edge white line position at 9879 eV.

Experimental and calculated reflectivities at $9850 \mathrm{eV}, 9879 \mathrm{eV}$ and $9950 \mathrm{eV}$ for Sample 110 are compared in Figure 2. These results show that the effect of the interfacial Ta monolayer in this multilayer is negligible. Very different results are found for Sample 111 where the Ta monolayer is placed at the other interface in the multilayer as shown by the experimental and calculated reflectivities presented in Figure 3. These properties are explained by calculations of the standing wave ficlds in these multilayer as shown in Figure 4. The Ta layers lie at the nodes of the standing wave field in Sample 110 and at the antinodes in Sample 111. This results in a strong absorption at the $\mathrm{x}$-ray energies $8979 \mathrm{eV}$ and $9850 \mathrm{eV}$ for Sample 111. The important conclusion to be drawn here is that, for $\mathrm{x}$-ray optics, control of the structure of one interface set in a multilayer is critical to performance. In this case, it is the WC on $\mathrm{C}$ interface.

Since there is absorption in the Ta layer at $8979 \mathrm{eV}$ which significantly impacts reflectivity there must also be Ta fluorescence which is determined by the distribution of Ta at the interfaces. The Ta fluorescence was detected using a $2 \mathrm{~cm}^{2}$ solid state ion chamber placed above and parallel to the sample surfaces. In Figure 5 the standing wave fluorescence generated by $8850 \mathrm{eV}$ and $8979 \mathrm{eV}$ x-rays is shown as a function of the angle of incidence on the samples. At $8850 \mathrm{eV}$ the fluorescence from the two are essentially identical. At $8979 \mathrm{eV}$ the signal from Sample 111 is clearly larger and less affected by primary extinction as expected from the reflectivity data. Modeling of this SWF data enables experimental measurement of the distribution of the Ta normal to the interfaces in the multilayer. Additionally, the magnitude of the SWF signals in these experiments indicates that very small interfacial 

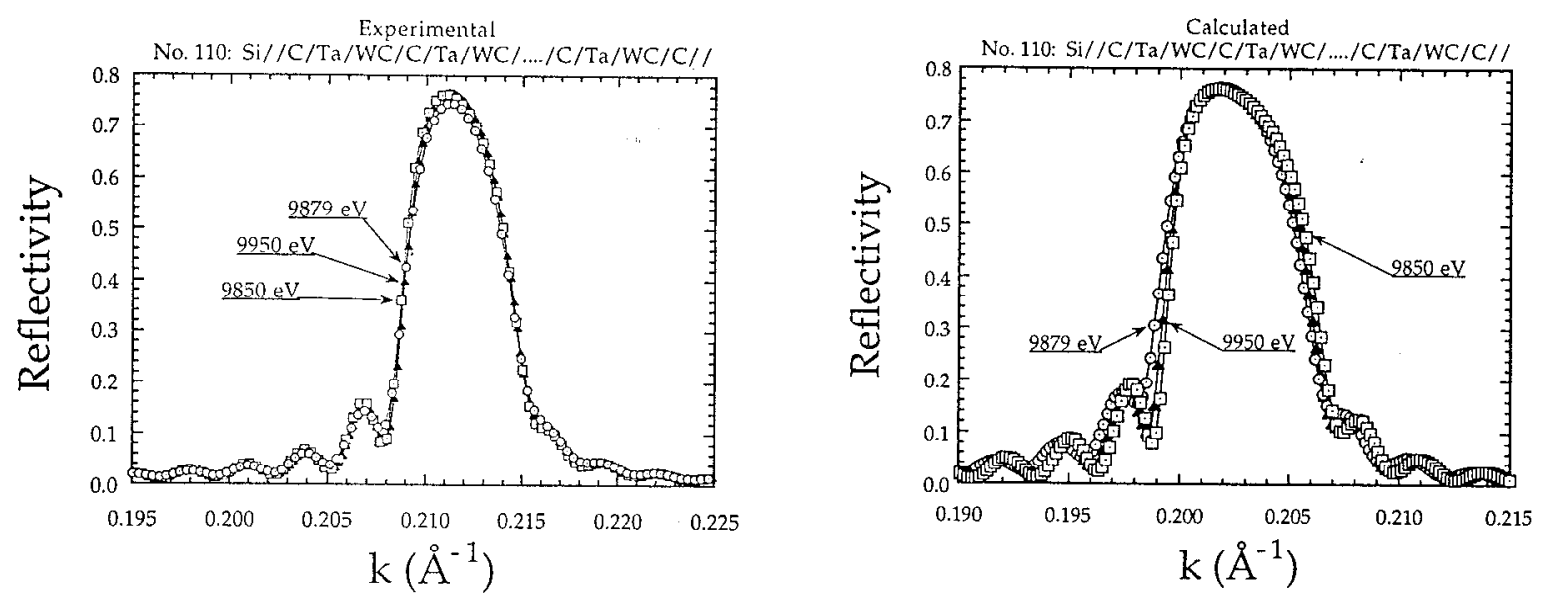

Figure 2 Experimental and Calculated reflectivities at $9850 \mathrm{eV}, 9879 \mathrm{eV}$ and $9950 \mathrm{eV}$ for Sample 110 are compared. The Ta interfacial monolayer has essentially no effect on the reflectivity when at the WC on C interfaces.
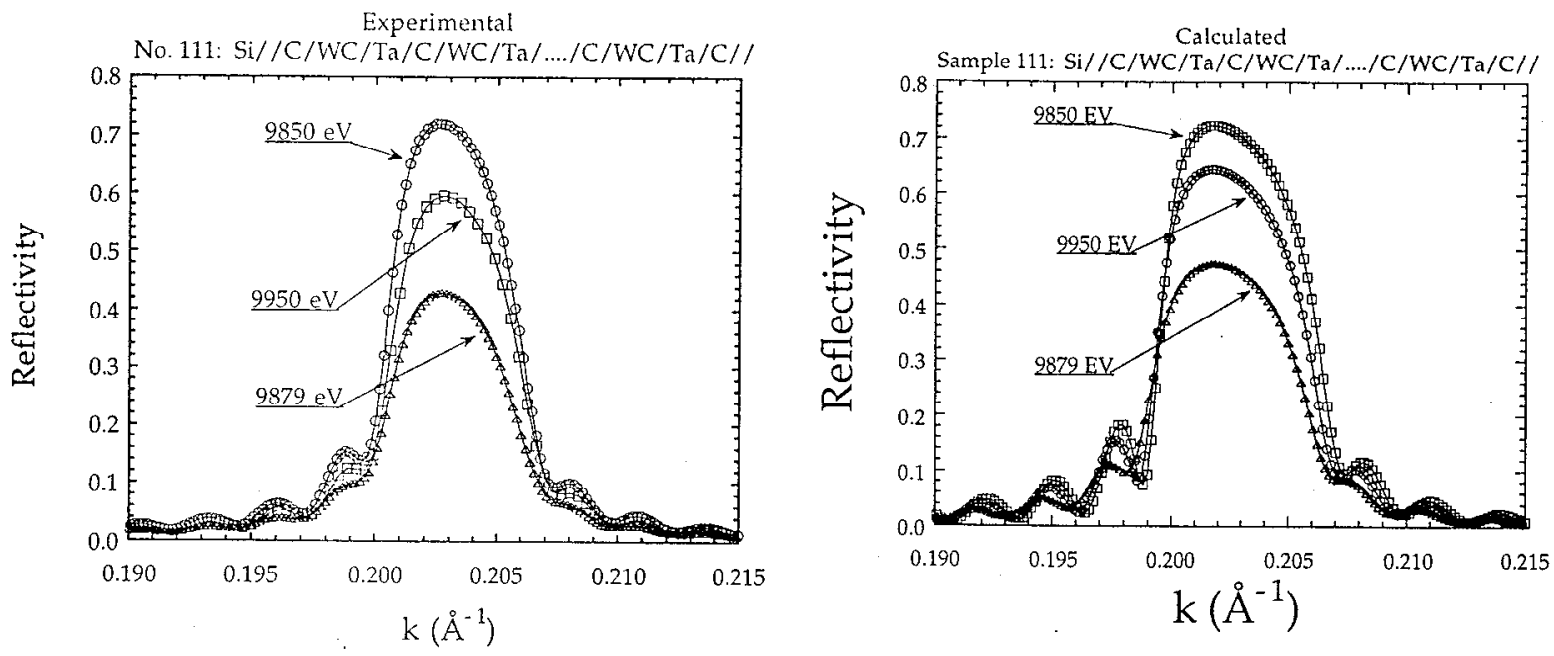

Figure 3. Experimental and calculated reflectivities for Sample 111 showing the impact on the reflectivity due to the Ta monolayer at the $\mathrm{C}$ on WC interfaces. This Ta position is at the antinode of the standing wave in the multliayer where an absorber or an imperfection would have the largest effect on the reflectivity.

concentrations can be delected and used to study interface atomic arrangements and electronic structure. This approach can be extended to fluorescence studies in the soft x-ray domain at energies less than $3 \mathrm{keV}$.

\section{DISCUSSION AND CONCLUSIONS}

The primary conclusion from these experiments is that the perfection of a specific set of interfaces in XR, SXR and EUV multilayers is crucial to their performance. In the work presented here an absorber, Ta, was applied as a representative imperfection at multilayer interfaces. Absorption in the Ta layers acted as the "imperfection" enabling the role of imperfection position in the multilayer to be demonstrated. Just as interesting is the potential that extrapolation of these observations to the study of buried interfaces holds. In this case the approach would be to decrease the concentration of the "marker element" to minimize its effects on the nature of the interfaces in the multilayer.

Calculations for multilayers with $2.3 \AA$ ( 1 monolayer[ml]), $1 \AA(0.43 \mathrm{ml}), 0.23 \AA(0.1 \mathrm{ml}), 0.1 \AA(0.023 \mathrm{ml})$ and $0.023 \AA(0.01 \mathrm{ml})$ of Ta at the interfaces were performed and showed that the effect of 0.01 monolayers is to 
decrease the reflectivity by approximately $0.4 \%$. The effect of 1 monolayer of Ta is to decrease the reflectivity by about $30 \%$. The decrease in Ta absorption at 0.01 monolayers is therefore about a factor of 75 . As stated earlier the
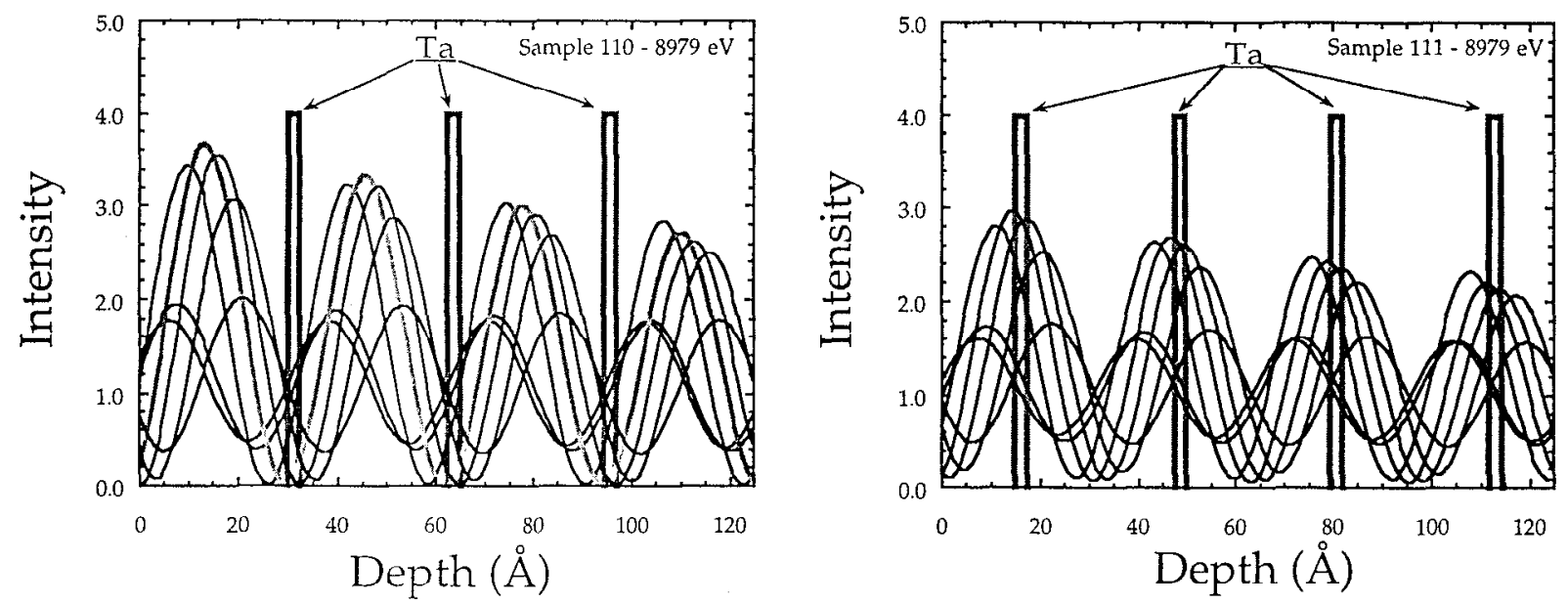

Figure 4. Calculated standing wave fields (SWF) in Samples 110 and 111 at the Bragg peak are shown. The Ta layers in Sample 110 fall at the nodes of the SWF thus minimizing their impact on the reflectivity. In contrast, the Ta layers in Sample 111 fall at the antinodes of the SWF maximizing their impact on reflectivity.

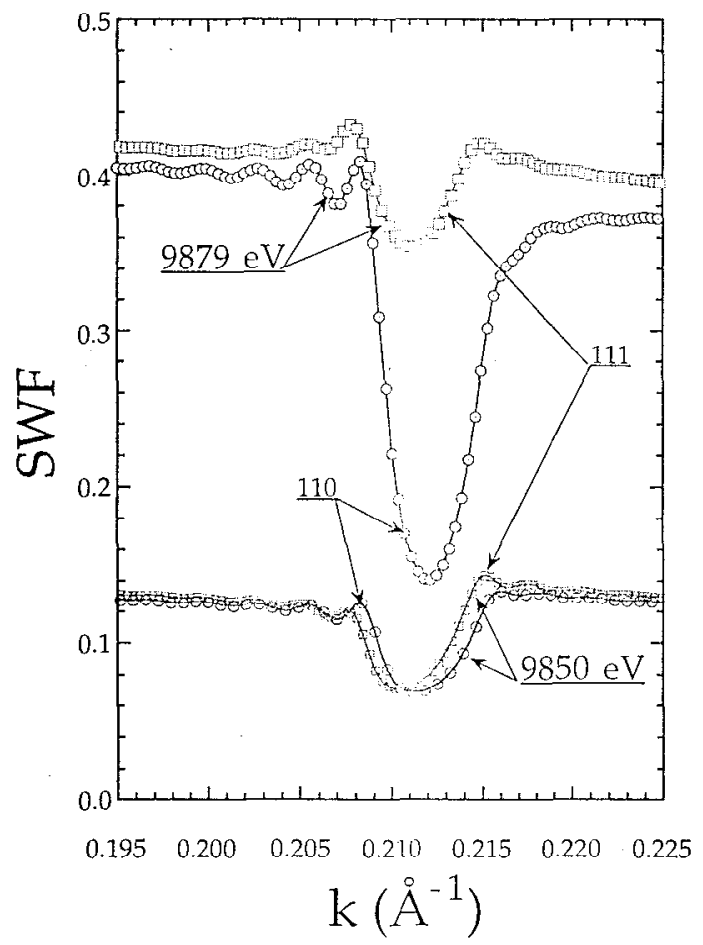

Figure 5. Experimental standing wave fluorescence intensities for No's 110 and 111 at $\mathrm{x}$-ray energies of $9850 \mathrm{eV}$ and $9879 \mathrm{ev}$ are shown as a function of $\mathrm{k}(=$ $4 \pi \sin \theta / \lambda)$. The SWF at $9850 \mathrm{eV}$ is essentially the same for $9850 \mathrm{eV}$ x-rays. At $9879 \mathrm{eV}$ No. 110 shows strong primary extinction on the Bragg peak as expected for an efficient structure. No. 111 exhibits a strongly attenuated primary extinction that indicates there is a strong loss mechanism operating at $8979 \mathrm{eV}$ - absorption in the Ta layer.

detector used had an area of $2 \mathrm{~cm}^{2}$. The detector area can be increased by a factor of 50 to $100 \mathrm{~cm}^{2}$ so that the fluorescent signal from the 0.01 monolayer Ta structure will be greater than $25 \%$ of that from the 1 monolayer Ta 
structure. This demonstrates that the "interfacial marker atom" approach can yield detailed atomic arrangement information at interfaces, chemical reaction data at interfaces at the atomic level and potentially, local electronic band structure characterization at interfaces. This exploratory modeling only applies to x-ray energies above $2 \mathrm{keV}$. Calculations of the reflectivity of a $\mathrm{WC} / \mathrm{C}$ multilayer at $1 \mathrm{keV}$ with 1 and 0.1 monolayer of copper at the interfaces were also performed. These calculations predicted a $6 \%$ absolute reduction in reflectivity at 1 monolayer $\mathrm{Cu}$ and $0.8 \%$ at 0.1 monolayer. This again indicate that the "interfacial marker element" technique will be effective in the soft $\mathrm{x}$-ray regime $(0.4$ to $2.0 \mathrm{keV})$.

The calculations directed to the exploration of the breadth of application of the "interfacial marker layer/interfacial marker element" technique have demonstrated the feasibility of the application of these approaches to the experimental study of the atomic level nature of atomic arrangements at interfaces, of chemical interactions at interfaces and potentially of electronic band structure effects at interfaces. Electronic band structure effects will be accessible for the transition elements through study white lines at their $L_{3,2}$ edges as the strength of these edge features is directly related to the $d$ band occupancy ${ }^{11}$ in the 4th (SXR), 5th ( SXR and XR) and 6th (XR) rows of the Periodic Chart. These opportınities are a direct consequence of the development of multilayer synthesis technology over the past two decades. This technology has made possible the conceptulization of monolayer scale control of composition in macroscopic samples. The world wide availability of intense scanning monochromatic x-ray and soft $\mathrm{x}$-ray sources at synchrotrons such as the Stanford Synchrotron Radiation Laboratory is also clearly crucial as such experiments would not be feasible without such facilities.

Therefore, the "interfacial marker layer/interfacial marker element" technique has the clear potential to yield atomic level experimental data on the nature of interfaces in solids. Such results will be valuable to theorists in that they will provide a basis for existing and to be developed formalisms. I note that at the lowest interfacial marker element concentrations ( $<0.01$ monolayer) this "experimental marker element" approach is a heterogeneous analogy to the homogeneous embedded atom ${ }^{12}$ theoretical methodology and, in principle, should provide a sound testing ground for that methodology.

\section{ACKNOWLEDGMENTS}

This work was performed under the auspices of the U.S. Department of Energy by the Lawrence Livermore National Laboratory under Contract No.W-7405-ENG 48 . UC-National Laboratory Participating Research Team facilities (BL 10-2) at the Stanford Synchrotron Radiation Laboratory (SSRL) were applied in this study. SSRL operates under the support of the U. S. Department of Energy.

\section{REFERENCES}

1. P. Dhez and C. Weisbuch, Eds., Physics, Fabrication and Applications of Multilayered Materials, Plenum Press, New York (1988).

2. Ph. Houdy, "Kinetic Ellipsometry Applied to Soft X-ray Multilayer Growth", Revue Phys. Appl. 23, 1653-1659, (1988).

3. M. Yamamoto and T. Namioka, "In Situ Ellipsometric Study of the Optical Properties of Ultra Thin Films", Appl. Opt. 31, 1612-1621, (1992).

4. J. Slaughter, P. Kearney and C. Falco, "Characterization of Pd-B, Ag-B and Si-B Interfaces", Proc. SPIE 1547, 71-79, (1991).

5. W. M. Stobbs, "Techniques for Characterizing Artificial Layer Structures Using the Fresnel Method", in Multilayers: Synthesis, Properties and Non-Electronic Applications, T. W. Barbee, Jr., F. Spaepen and L. Greer, Eds., MRS Sym. Proc. 103, 121-131,(1988).

6. W. C. Shih and w. M. Stobbs, "Measurement of Roughness of W/Si Multilayers by the Fresnel Method", Ultramicroscopy 32, 219-239, (1990).

7. E. Spiller, Soft X-ray Optics, SPIE Optical Engineering Press (1994).

8. T. W. Barbee, Jr., "Multilayers for X-ray Optics", Opt. Eng. 25, 898-915, (1986).

9. E. Spiller, "Characterization of Multilayer Coatings by X-ray Reflection", Revue Phys. Appl. 23, 1687-1700, (1988).

10. T. W. Barbee, Jr. and W. K. Warburton, "Evanescent and Standing Wave Fluorescence from a Multilayer Structure", Materials Letters 3, 17-25, (1984).

11. D. H. Pearson, C. C. Ahn and B. Fultz, Phys. Rev. B47, 8471-8478 (1993).

12. M. S. Daw, S. M. Foiles and M. I. Baskes, Mat'ls. Sci. Rpts 9, 251-310 (1993) 